\title{
A Novel Approach Based on Fluid Dynamics for On-Site Safety Assessment
}

\author{
Mei Liu ${ }^{\circledR a}$, Heap-Yih Chong ${ }^{\circledR b}$, and Pin-Chao Liao ${ }^{\circ}$ \\ aSchool of Urban Economics and Management, Beijing University of Civil Engineering and Architecture, Beijing 100044, China \\ ${ }^{b}$ School of Design and the Built Environment, Curtin University, Perth, WA 6845, Australia \\ 'Dept. of Construction Management, Tsinghua University, Beijing 100084, China
}

\section{ARTICLE HISTORY}

Received 9 June 2020

Revised 30 September 2020

Accepted 6 December 2020

Published Online 12 February 2021

\section{KEYWORDS}

Fluid dynamics

Safety assessment

Risk evaluation

Management

Construction

\begin{abstract}
Construction safety assessment is a major component of safety management in construction projects. However, thus far, most assessment studies have focused on either cross-sectional or longitudinal performance. This study aims to develop a proactive and preliminary safety assessment method using a fluid dynamics (FD) approach by integrating longitudinal contractor performance with cross-sectional safety conditions. To this end, an FD framework was first developed using three processes: (a) identifying the connections between FD principles and safety analogs, (b) incorporating the Darcy-Weisbach equation into the framework, and (c) modifying FD equations for construction safety assessment. Subsequently, two case studies were investigated, and the results obtained were compared and verified with one existing assessment results. The results indicate that the state of safety at a construction site incorporates both the occurrence of hazards and conservation of energy. Thus, the proposed FD approach can be used to preliminarily assess and predict safety conditions by combining safety-related measures with the dynamic characteristics of construction processes. The approach considers a comprehensive range of indicators and parameters, which enables the comparison of safety performance between projects or assessment periods by independently changing parameters.
\end{abstract}

\section{Introduction}

Traditionally, improvements in safety have been driven by historic data. Site safety developments have been reactive and not proactive. That is, the occurrence of a serious injury/fatality, or a high accident rate, initiates investigation to identify and prevent further incidence. While frequency of injury/fatality in construction is high with respect to other industries, actual frequency of accidents is not great enough to accurately model and make predictions based on historic data (Hopkins, 2009), particularly when assessing smaller scale projects. Although monitoring this data is necessary and will continue to play a role in safety management, these are defined as lagging indicators. The historic nature of a lagging indicator means it cannot accurately reflect the current status of safety management systems and environment (Grabowski et al., 2007; Hinze et al., 2013). Lagging data does not indicate actions taken to address safety concerns. Therefore, a need exists for new proactive methods of risk management and warning systems to be developed for the construction industry.

The importance of improving safety performance in construction has prompted a shift in the direction of safety management studies from reactive to proactive strategies. Approaches to improve unsatisfactory safety performance include identification, benchmarking, and analysis of leading indicators as an alternative to traditional risk assessment and safety performance monitoring (Guo and Yiu, 2016; Versteeg et al., 2019; Hallowell et al., 2020). Currently, proactive safety strategies and management practices in construction projects still primarily rely on physical evaluations and accident reporting (Hinze et al., 2013). Safety checklists and basic probabilistic hazard assessments are used to control risks, which encompass various safety indicators, such as audit and organizational performance (Rajendran, 2013; Shea et al., 2016; Gunduz, 2018), frequency of pre-start meetings and inspections (Lingard et al., 2017), safe and unsafe conditions and activity 
Table 1. Main Assessment Methods

\begin{tabular}{lll}
\hline Method classification & Method properties & Main representative method \\
$\begin{array}{l}\text { Subjective evaluations based on expert } \\
\text { knowledge }\end{array}$ & Subjective evaluation & $\begin{array}{l}\text { Peer review, expert review, Delphi method, investigation, and research } \\
\text { method }\end{array}$ \\
$\begin{array}{l}\text { Objective evaluations based on statistical } \\
\text { data }\end{array}$ & Objective evaluation & $\begin{array}{l}\text { Correlation coefficient test, entropy method, Bayesian method, principal } \\
\text { components analysis, and cluster analysis }\end{array}$ \\
$\begin{array}{l}\text { Comprehensive evaluations based on } \\
\text { system model }\end{array}$ & $\begin{array}{l}\text { Comprehensive } \\
\text { evaluation }\end{array}$ & $\begin{array}{l}\text { Fuzzy-AHP, fuzzy fault tree, artificial neural network, system dynamics, } \\
\text { TOPSIS, fuzzy-grey model, grey relationship analysis, matter-element } \\
\text { method, set pair analysis (SPA), fuzzy set, reasoning, and simulation }\end{array}$ \\
\hline
\end{tabular}

observations (Fang et al., 2004; Xia et al., 2018), infringements, hazards, and hazards rectified (Lingard et al., 2017). The inclusion of real-time management performance has also been proposed, which can have a significant influence in proactive warning of safety outcomes (Sheehan et al., 2016; Thurston and Glendon, 2018; Wang et al., 2020). Innovative assessment approaches (e.g., fuzzy AHP and TOPSIS) that combine multiple safety-related measures have been proposed (Kamardeen, 2009; Hosny et al., 2013; Esmaeili et al., 2015; Kumar and Garg, 2016; Lingard et al., 2017; Mohammadi et al., 2018); however, these approaches cannot estimate the changing safety environment because they do not consider the dynamic characteristics of construction processes, such as the life cycle of a project (Cooke, 2010; Mahmoud et al., 2019; Hallowell et al., 2020).

This study aims to develop a preliminary and proactive safety assessment method using the Fluid Dynamics (FD) approach by integrating longitudinal contractor performance with cross-sectional safety conditions. First, the usual risk evaluation methods and the application of FD were reviewed. Second, the analogy and FD parameters were examined to align with safety analogs. Third, an FD framework was developed based on modified equations for construction safety assessment. Subsequently, the developed framework was examined via two case studies in China. Finally, the assessment results obtained were compared with the results of one existing original assessment conducted during the course of the projects.

\section{Literature Review}

\subsection{Dynamic Characteristics of the Construction Processes and Current Approaches}

Typical risk evaluation methods primarily adopt reactive indicators of construction safety performance by characterizing risk probability and risk severity in the presence of a hazard or a dangerous situation (Hou and Zeng, 2016). They focus on identifying the relationship between risk factors and safety performance to obtain key risk factors (Alruqi et al., 2018; Yu et al., 2018; Ayhan and Tokdemir, 2020). However, these reactive indicators exhibit certain limitations (Shea et al., 2016; Sheehan et al., 2016; Robson et al., 2017). For example, they have a low predictive value as they are relatively constant during a project and neglect constant changes to the physical site, and human or management factors (Cooke, 2010; Han et al., 2014; Xia et al., 2018). In contrast, active indicators have a high predictive value and hence can highlight short-term changes in safety (Hinze et al., 2013). Proactive actions are necessary to prevent accidents by evaluating on-site hazards and their dynamic risk systems (Hopkins, 2009; Emmanuel et al., 2016; Guo and Yiu, 2016). This proactive approach could help in improving the construction safety assessment by predicting on-site uncertainties and changes.

Table 1 summarizes the extant common assessment methods. Among these methods, subjective evaluations are commonly used to determine the weighting coefficients of factors based on scores from expert interviews and questionnaires (Rubio-Romero et al., 2018). This approach does not require on-site records, as collecting several consecutive data records related to management activities is difficult. However, the method is subjective and does not reflect the objective attributes and value orientation of the evaluated objects (Deng et al., 2017).

In contrast, objective assessment methods based on statistical data can be used to calculate the weighting of safety indicators (Christodoulou, 2010; Long et al., 2016). A few studies used measured results as decision variables to determine the weight of indices, such as correlation coefficients (Alruqi et al., 2018). When no decision variable exists, the key factors can be found by comparing the differences and correlations between the indicators, such as principal component analysis, cluster analysis, and entropy-based method (Deng et al., 2017).

Fuzzy AHP, TOPSIS, and other innovative assessment approaches that combine multiple safety-related measures have been proposed (Kamardeen, 2009; Hosny et al., 2013; Esmaeili et al., 2015; Kumar and Garg, 2016; Lingard et al., 2017; Mohammadi et al., 2018). (Pereira et al., 2018) proposed a conceptual approach that used case-based reasoning and simulation modeling to update safety-related measures at specified time intervals by allowing the assessment of safety performance to replicate the life cycle of the project. Moreover, a few studies defined and included the dynamic characteristics of a construction project to investigate managerial performance by considering management factors through system dynamic modeling (Chritamara et al., 2001; Mohamed and Chinda, 2011). However, changes in these factors have a limited ability to reflect dynamic situations at construction sites. For example, consider "hazard workers working at height without a fall-arrest system"; if safety inspectors can rapidly remedy this unsafe situation, the possibility of fall accidents can be considerably eliminated. Therefore, a comprehensive 
safety assessment system should consider the dynamic characteristics of the construction processes, given their potential to provide early warning signs of safety failure and their ability to proactively drive safety improvements (Fang et al., 2004; Grabowski et al., 2007).

\subsection{Application of FD to Alternate Domains}

Fluid mechanics has been considered in various studies to address the dynamic characteristics and continuing processes in construction projects due to its flexibility and accuracy in fluid (uncertainty) simulations and quantitative predictions. In fact, this method has been effective for solving problems in diverse domains (Mishra, 2015; Seminara et al., 2020), such as traffic transportation (Lighthill and Whitham, 1955; Richards, 1956), ecology (Jones et al., 2018), supply chain inventory analysis (Kachani and Perakis, 2006), and manufacturing systems (Wiendahl et al., 2002; Efthymiou et al., 2009). These studies have provided insights into dynamic phenomena that exhibit characteristics similar to those of fluid mechanics. The on-site hazard and risk system is dynamic (Nima et al., 2014). However, limited empirical studies have been conducted on applying fluid dynamics (FD) for construction safety assessment.

Analogical argument is the inference of similarities between systems, particularly based on other shared similarities. Analogies between domains may refer to objects, properties, relations, and functions as a basis of reasoning (Bartha, 2013). To some extent, analogies can justify the formulation and pursuit of new theories. However, even a weak analogy, presented while poorly arguing for a conclusion, indicates some degree of plausibility and supports further investigation of a hypothesis (Bartha, 2013). We present an analogical argument that the similarities identified between the domains in terms of movement and force interactions suggest that FD principles may have value in assessing construction safety performance.

Bernoulli's equation and the Darcy-Weisbach equation are commonly used to describe fluid flow through different media. Bernoulli's equation (Darrigol and Frisch, 2008) is a conservation of energy equation based on Bernoulli's principle, which considers the pressure, kinetic, and potential energy of a flowing fluid. The Darcy-Weisbach equation (Manning and Richard, 1991) estimates the head loss or pressure loss due to friction along a given length of pipe, given the average velocity of an incompressible fluid. The mathematical principles that govern FD contribute to the development of knowledge outside the domain. FD analogies, such as Maxwell's, continue to be used in electromagnetics, whereas several similar analogies have been extended to other domains and now have been established in these areas. Vehicle traffic flow models are typically developed using FD principles (Lighthill and Whitham, 1955; Richards, 1956; Tosin and Zanella, 2019; Guo et al., 2020), and several traffic-related theories are analogous to fluid-related theories (Peng, 2013; Ma et al., 2018; Zhao et al., 2018). Pedestrian movement studies have also adopted FD analogies as a foundation for modeling (Qiu et al., 2018; Sun, 2018). FD models have been utilized in an ecological context to estimate species movement rates across terrestrial landscapes (Feliciani and Nishinari, 2018), supply chain inventory analysis (Kachani and Perakis, 2006), and manufacturing systems (Wiendahl et al., 2002; Efthymiou et al., 2009).

These examples illustrate the versatility and effectiveness of applying mathematical models and theories to domains outside the one in which they were established. Analogies have not validated these applications, rather, concepts born from analogies have been validated through subsequent investigations. Similarly, in this study, we use analogy only as a tool to develop a model. Only through practical applications will the methodology be validated.

\section{Development of FD Framework}

Three main processes were used for the development of the FD

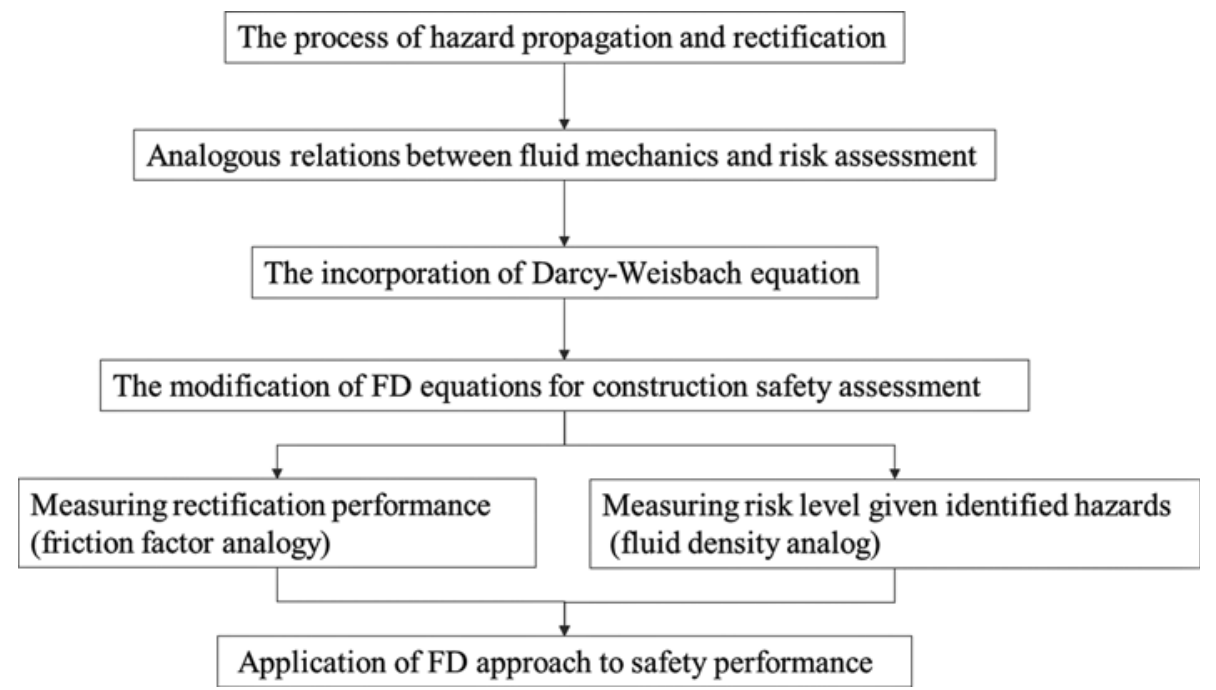

Fig. 1. Overall Methodological Steps of the Framework Development 
framework: (a) linkage between FD principles and safety analogs, (b) incorporation of the Darcy-Weisbach equation, and (c) modification of FD equations for construction safety assessment. Fig. 1 illustrates the overall methodological steps of the development of the proposed framework through the principles of basic fluid flow.

\subsection{Linking FD Principles to Their Safety Analogs}

The method of assessment was developed based on a relationally focused analogy between safety risk and FD. The safety assessment is based on hazard propagation and rectification, as illustrated in Fig. 2. We consider the development of a set of hazards, $A-E$, at time $t 1$, and the corresponding risk level, $S$, due to these hazards (step 1). As the risk flows through the project during the assessment period, hazards $B$ and $C$ are rectified such that the real risk level is reduced to $S^{\prime}$ at $t 2$ (step 2). Next (step 3), we illustrate the resistance to the flow of hazards through the site due to the rectification performance of the management team. The resistance then contributes to a reduction in the energy of risk during the assessment period, the difference value $\Delta=W_{f}$

Next, we consider the relationship between the frequency of hazard occurrence and the total risk level. When the ratios of the total risk level to the project construction area and other factors are constant, the framework favors hazards of lower consequences over more severe hazards, given the location of $v$, the hazard transfer rate in the numerator, and the density in the denominator. Thus, considering two projects with the same absolute risk level and project areas, the project with the higher number of low consequence hazards will have a higher score in the safety assessment than a project with fewer but more severe hazards. We consider this a significant factor in assessing project safety.

Rectification is specific to hazards and is defined as the performance of the construction-safety management team in resisting the flow of hazards through the site. The higher is the friction within the system, the higher is the pressure drop. Similarly, a larger hazard rectification force generated by management results in a more significant safety risk reduction. In this study, we consider rectification performance as the only force that resists safety risk. However, other factors influence the likelihood of the occurrence of an accident due to hazards, such as safety culture and training. These other factors are analogous to friction due to pipe wall roughness. By considering only rectification performance, we assume that the project is represented as a smooth-walled pipe and resistance due to this element is negligible.

Therefore, we suppose that hazards on construction sites "flow" through time as fluid flows through a pipe. In this context, project hazards can be thought of as individual particles of the fluid, and the risk level is related to the fluid pressure as it moves through the pipe. The opposing frictional forces within the pipe system are analogous to the opposing force of safety management, and hence, help resist the flow of risk through a project by hazard identification and rectification. The responsiveness of management to eliminate risks through inspection and rectification is a force that counteracts unsafe site conditions and actions that contribute to accidents.

The FD principles have considerable potential in assessing construction safety performance based on analogical arguments (Table 2).
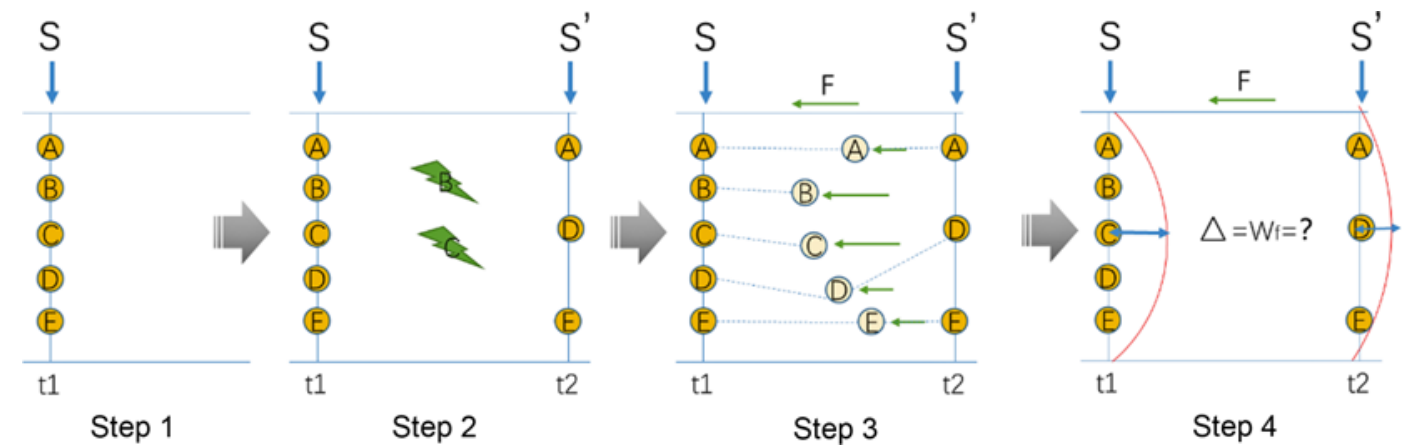

Fig. 2. Process of Hazard Propagation and Rectification

Table 2. Horizontal and Vertical Analogous Relations between Fluid Mechanics and Risk Assessment

Fluid mechanics (S)

Risk evaluation $(\mathrm{T})$

Flow of fluid particles through a charged pipe is continuous

Friction forces resist pipe flow, resulting in dissipation of energy within the system.

Evaluated by fluid dynamics principles (Bernoulli's Principle, Darcy-Weisbach)
Known Similarities

$\leftarrow$ Horizontal relations $\rightarrow$ Hazards and risks on a construction site are ongoing Rectification efficiency of management resists hazards, resulting in dissipation of project risks.

Inferred Similarities 
Table 3. Relationship between FD Terms and Their Project Risk Analogues

\begin{tabular}{llll}
\hline Parameter & Symbol & Unit & Risk analogue \\
\hline Friction factor & $\lambda$ & - & Resistance to hazard flow, rectification performance \\
Velocity of fluid & $v$ & $\mathrm{~m} / \mathrm{s}$ & Risk transfer rate/number of project risks per evaluation period \\
Viscosity of fluid & $\mu$ & $\mathrm{m} / \mathrm{s}$ & Rectification coefficient/ rectification affection on hazard \\
Density of fluid & $\rho$ & $\mathrm{kg} / \mathrm{m}^{3}$ & Density of risk, risk consequence per unit area \\
Pipe diameter & $D$ & $\mathrm{~m}$ & Area of risk assessment \\
Length of pipe section & $L$ & $\mathrm{~m}$ & Time period on assessment \\
Pressure, at ref. point $x$ & $p_{x}$ & $\mathrm{~Pa}$ & Risk level across the project for a single time period \\
Energy loss & $W_{f}$ & $\mathrm{~J}$ & Comprehensive safety performance score \\
\hline
\end{tabular}

\subsection{Applying the Darcy-Weisbach Equation to the Construction Safety Assessment}

Having drawn an abstract analogy between fluid flowing through a pipe and risks through a project site, an established mathematical model was considered in the FD framework. The Darcy-Weisbach equation contains a dimensionless friction factor, known as the Darcy friction factor or resistance coefficient. Both the head loss and energy loss forms of the equation are as follows:

$$
\begin{aligned}
& h_{f}=\lambda \frac{L}{D} \frac{v^{2}}{2 g}, \\
& W_{f}=\lambda \frac{L}{D} \frac{v^{2}}{2},
\end{aligned}
$$

where $h_{f}$ is the head loss, $W_{f}$ is the energy loss, $L$ and $D$ are the pipe length and diameter, respectively, $v$ is the fluid velocity, $g$ is the gravitational constant, and $\lambda$ is a coefficient of friction.

The friction, or resistance factor, $\lambda$, is dependent on the characteristics of the pipe (diameter D and roughness height $\varepsilon$ ), characteristics of the fluid (its kinematic viscosity), and the velocity of the fluid flow. The equation has been approximated with high accuracy for certain flow types and may be evaluated based on various empirical relations or published charts. For laminar (smooth) flows, the friction factor can be determined by Poiseuille's law, which is derived from an exact classical solution for fluid flow:

$$
\lambda=\frac{64}{R e},
$$

where $R e$ is the Reynolds number, which is equal to

$$
R e=\frac{D v \rho}{\mu},
$$

where $D$ is the pipe diameter; $v$, the fluid velocity; $\rho$, the density coefficient of the fluid; and $\mu$, the viscosity of the fluid.

Therefore, the energy loss of the fluid in a laminar flow in a circular straight pipe is

$$
W_{f}=\frac{64}{R e} \frac{L}{D} \frac{v^{2}}{2}=\frac{64}{\frac{D v \rho}{\mu}} \frac{L}{2} \frac{v^{2}}{2}=\frac{32 \mu L v}{D^{2} \rho} .
$$

We now relate each of the terms in FD (Eqs. (1) and (5)) to construction safety inspection and define their analogs (Table 3), ensuring the compatibility of concepts between domains.

The energy loss, $W_{f}$, is dependent on the characteristics of the fluid and the system through which it is moving. We propose that this parameter and equation can be modified and applied to safety performance assessment. Fluid density, $\rho$, and its velocity, $v$, can be related to the propagation of hazards on site. Diameter, $D$, and length, $L$, are basic characteristics of the system and can easily be transferred to the project context. However, to accept the analogous application of these fluid principles, we must consider that the resistant force, $\mu$, is analogous to hazard rectification only in terms of its influence on the dependent variable. That is, the physical phenomena of fluid viscosity share no physical attributes with hazard rectification; however, both produce resistant forces, which reduce the energy and risk level of their respective systems.

The selection of this FD for construction safety assessment is based on the following assumptions: 1) The flow of risk is represented as a laminar flow; 2) The speed of the hazard flow is determined solely by the frequency of hazard occurrence and rectification performance within a time period; and 3) The area of the entire project, that is, the diameter of the pipeline, does not change during construction.

\subsection{Modifying the FD Equations for Construction Safety Assessment}

Now, we configure the energy loss Eq. (6) for construction safety assessment and determine the plausibility of the framework by ensuring that the functions of the parameters within the framework are coherent. We demonstrate the application of the modified FD equation in case studies.

First, we define the cross-sectional area, $A$, of the construction site, which can be found in basic project information material. Then, the diameter, $D$, is

$$
D=\frac{4 A}{\pi}
$$

We consider the pipe length, $L$, to be the duration of a single assessment (defined as one month in this study). Fluid velocity, $v$, can be considered the risk transfer rate, or the number of risks per assessment period, and $\rho$ is the density of risk, which is equal to the risk level per unit area due to hazards observed. 
The final pressure loss due to friction across the system is analogous to risk reduction due to the rectification performance for the project over a single period. Therefore, energy loss is considered a comprehensive safety assessment index. The index value for a given project for a specified time period can be calculated as follows:

$$
W_{f}=\frac{2 \mu L v \pi^{2}}{A^{2} \rho} \text {. }
$$

Considering the reconfigured FD Eq. (7), the relationship between the factors and their influence on the safety assessment score are clearly expressed. First, we can see the interaction between the risk level and rectification performance on the safety assessment index. When all other parameters are equal, an increase in rectification performance, $\mu$, will increase safety index value, whereas a decrease in $\mu$ will decrease the safety index value. Given the situation in which two physically identical projects are subject to the same hazards in an assessment period, the management team's ability to respond to hazards is considered when evaluating the comprehensive safety level of each project. This is consistent with our understanding of the role of management and rectification performance in mitigating risks. Conversely, the density of risk, $\rho$, is inversely proportional to the safety index. An increase in the density of risk produces a lower safety assessment. Finally, we also observe that this framework considers project construction area as a contributor to safety level. When all other parameters are equal, the safety assessment score generated by this framework will be lower for a larger project. Larger projects are likely to be more complex; involve several employees, plant movements, and parallel activities; get subcontractors to be more involved; and have less direct supervision from management and a high likelihood of unidentified hazards (López-Alonso et al., 2013; Feng et al., 2015).

\section{Case Study}

\subsection{Data Sources}

Data were collected from two construction projects in Shandong province, China, between June 2016 and January 2018. Weekly checks were conducted by third-party safety inspectors. The safety experts employed by the third-party were engaged in the construction industry for $10-30$ years and had vast experience in construction safety management. The safety scores were collated to provide a monthly assessment. In the safety inspection process, safety inspectors followed the guidelines documented in the Standard for Construction Safety Inspection JGJ59-2011 (2011). A standard list of safety performance indicators was used to record details of nonconformance, location, rectification deadlines for new hazards, and rectification status of previously identified hazards. For critical hazards, inspectors supervised the construction workers to conduct immediate rectification or prescribed a deadline for rectification (for example, 3 days). The status and date of hazard rectification were also monitored and recorded. A total of 1963 hazard items and rectification statuses were logged across the seven projects between June 2017 and December 2017 and used as the database for this analysis. Inspection data were provided by project owners through the safety management platform and shared with the academic research team. The proposed FD approach was applied and tested in these projects to demonstrate the application process and functionality as a safety assessment tool.

\subsection{Measuring Rectification Performance (Friction Factor Analogy)}

The friction factor analogy adopted within the proposed assessment method is a measure of management performance in rectifying hazards identified within the site during an assessment period. The factor should reflect the overall rectification performance by considering and differentiating the resolution state and timeliness of individual hazards. Thus, three rectification states were defined: 1) rectified on time, 2) overdue rectification, and 3) overdue non-rectification. The three classifications were based on rectification deadlines granted by the safety assessor conducting an inspection. The deadlines were decided by safety experts according to their professional experience. Seven potential

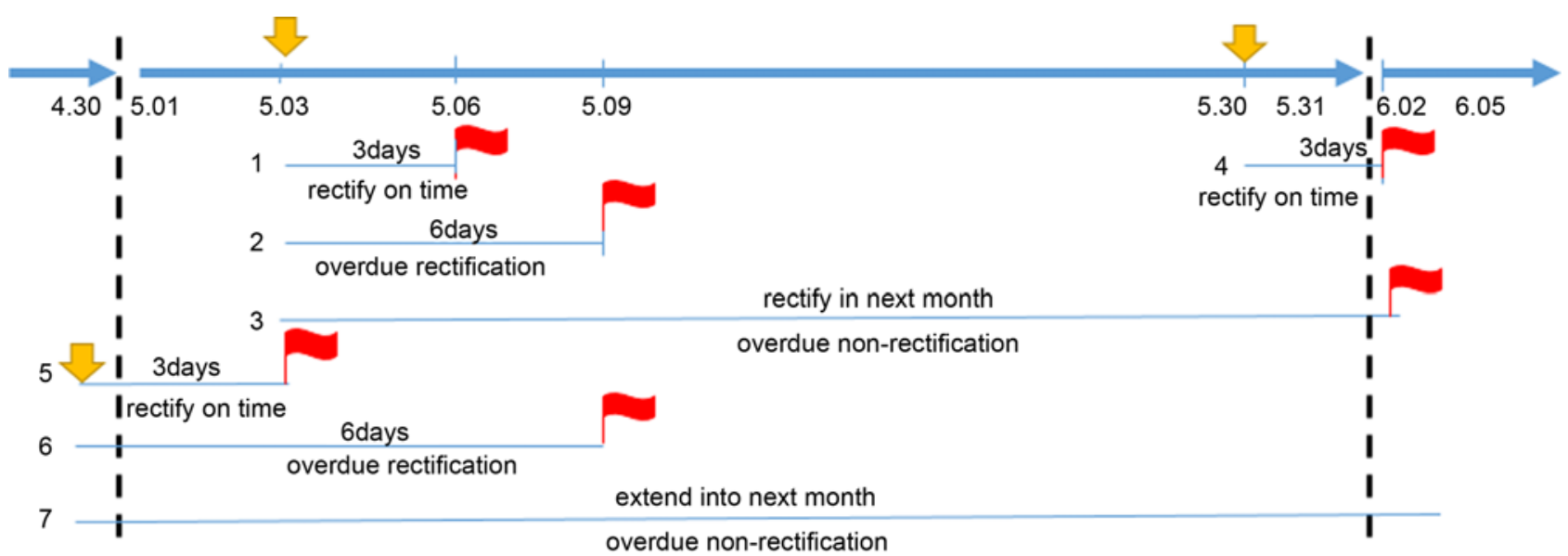

Fig. 3. Potential Rectification Scenarios Differentiated by the Rectification Performance Parameter 
Table 4. Classification of Rectification States for Rectification Scenarios in One Month

\begin{tabular}{|c|c|c|c|c|c|}
\hline Scenarios & Identified & Rectified & $\begin{array}{l}\text { Rectified within the } \\
\text { allowable period }\end{array}$ & Rectification states & Calculated in this month \\
\hline 1 & $\sqrt{ }$ & $\sqrt{ }$ & $\sqrt{ }$ & Rectified on time & $\sqrt{ }$ \\
\hline 3 & $\sqrt{ }$ & $\times$ & $x$ & Overdue non-rectification & $\sqrt{ }$ \\
\hline 4 & $\sqrt{ }$ & $\times$ & $\sqrt{ }$ & Rectified on time & $\sqrt{ }$ \\
\hline 7 & $x$ & $x$ & $\times$ & Overdue non-rectification & $\sqrt{ }$ \\
\hline
\end{tabular}

scenarios were identified based on the time of hazard occurrence, as shown in Fig. 3 and Table 4.

If a hazard was identified in an assessment month and rectified within the allowable period, it was allocated a "rectified on time" rating for that hazard for that month (scenario 1 and 4). If a hazard was rectified within the allowable time, but the hazard occurred again in the preceding month and was rectified accordingly in that month, its rating was allocated only to the month in which the hazard was identified (scenario 5). If a hazard was rectified in the assessment month, but rectification exceeded the allowable time (scenarios 2 and 6), irrespective of the month in which the hazard was identified, it was allocated a "late rectification" classification. In either situation, if a hazard remained unresolved at the end of the month, from either that month or an earlier period, and was overdue, that item was allocated an "overdue non-rectification" rating.

\subsection{Measuring Risk Level Given Identified Hazards (Fluid Density Analog)}

We adopted the standardized risk weighting allocated by the "Standard for Construction Safety Inspection" (Zhang, 2011) to quantify the severity of each hazard identified. Thus, we applied an entropy weighting method to determine the risk level, given the complete set of identified hazards. In information theory, an entropy weighting method derives a measure of the degree of disorder, or uncertainty, within a system based on available information (Li and Zhao, 2017). This method has been applied in risk assessment and safety assessment studies to determine objective weighting indices ( $\mathrm{Li}$ et al., 2011; Li and Zhao, 2017). While other methods of risk quantification could be used to derive this parameter, entropy was selected based on its consistency and ability to reduce bias and subjectivity associated with traditional probabilistic risk assessment.

\subsection{Application of the FD Approach to Safety Performance}

To apply the FD concept to the comprehensive safety assessment of construction projects, we adopted the modified energy loss Eq. (8) as the basis of assessment.

To assess the case projects, we defined a single assessment period, $L$, to be one month. Therefore, the hazard transfer rate, $\mathrm{v}$, was equal to the number of hazards per month. This information was obtained directly from the data records. As the frequency of site inspection varied depending on the month (four or five times per month), we normalized the data as the average number of hazards per inspection per month. The construction area, $A$, was obtained from the basic project information records.

The density of hazards, $\rho$, was then calculated as follows:

$$
\rho=\frac{S}{A},
$$

where $A$ is the project area and $S$ is the absolute risk level of all hazards without rectification, which can be calculated from the occurrence and seriousness of hazards. Here, we used an entropy weighting method to establish $S$. By considering the risk density, we allowed for incomplete checks and had the ability to include partial site inspection data.

Next, the rectification coefficient $\mu$, was determined via quantification of hazard rectifications per month. The rectification states were classified into three categories: rectified on time, overdue rectification, and overdue non-rectification (demonstrated in Fig. 3). The score of each category was calculated using the occurrence frequency and seriousness of hazards in each category. In sequence, we used the competitive cooperation negotiation model (Gao et al., 2018) to obtain the weight of each category. Finally, the rectification performance value per month was generated based on the score and weight.

According to the data simulation output (equidistance gradient) and the actual situations within construction, safety performance was divided into four grades: grade 1 (worst), grade 2 (poor), grade 3 (better), and grade 4 (best). The related thresholds were determined as $(-\infty,-1],(-1,0.5],(0.5,30],(30,+\infty)$, respectively.

By taking one of the projects in October 2016 as an example, the inspectors conducted four checks and identified 21 hazards. All hazards were rectified on time. The inspection area was $20,640 \mathrm{~m}^{2}$. The calculation results showed the hazard consequence $S$, equal to 0.1475 , and the rectification coefficient, $\mu$, equal to 0.0981 . Then, the energy loss as a comprehensive safety assessment index was calculated using Eq. (8) as follows:

$$
W_{f}=\frac{2 \mu L v \pi^{2}}{A^{2} \rho}=\frac{2 \times 0.0981 \times 1 \times(18 / 4) \times \pi^{2}}{2.0640^{2} \times(0.1475 / 2.0640)}=28.5960 .
$$

Thus, safety performance for this month was Grade 3 (better), 
indicating that although new hazards occurred at the construction site, most of them were controlled, such that the hazard rectification efficiency was relatively high, and the likelihood of accidents was relatively low.

\subsection{Comparing with Existing Assessment Method}

The proposed FD safety framework was compared with the existing assessment method used by the company. The original method (OM) was initially designed based on expert experience and was applied to the projects in these cases. The method was structured as follows. First, hazards were divided into three degrees - general, moderate, and major-according to their consequences. Second, according to the state of hazard rectification, the hazards were divided into rectified on time and overdue rectification. The different values for hazards were generated by experts, as listed in Table 5. Finally, based on the total score in one month, the assessment of safety performance was distinguished by four grades: grade 1 (worst), grade 2 (poor), grade 3 (better), and grade 4 (best), with the corresponding thresholds determined by experts as $[300,+\infty),[150,300),[50,150)$, and $[0,50)$, respectively.

For example, if there were two general hazards, which were all rectified on time, and another major hazard that was classified as overdue rectification, the safety performance score would be $2 \times 2+1 \times 100=104$. This would be assigned a rating of 3 (better), indicating that the occurrence of new hazards was generally controlled and rectified with efficiency.

Table 5. Value of Each Hazard Using Assessor's Existing System

\begin{tabular}{llll}
\hline Hazard value & $\begin{array}{l}\text { General } \\
\text { degree }\end{array}$ & $\begin{array}{l}\text { Moderate } \\
\text { degree }\end{array}$ & $\begin{array}{l}\text { Major } \\
\text { degree }\end{array}$ \\
\hline Rectify on time & 2 & 10 & 50 \\
Overdue rectification & 4 & 20 & 100
\end{tabular}

\section{Findings and Discussion}

\subsection{Comparison and Verification}

The energy loss as a comprehensive safety assessment index by the FD approach was obtained as summarized in Table 6 (e.g., Project 1 and Project 2). Based on the total score in Table 6, the assessment of safety performance was distinguished by four grades. Then, by comparing the results of the $\mathrm{OM}$ and the FD, the characteristics of the application of FD were analyzed from the aspect of risk assessment. Figs. 4 and 5 illustrate the safety

Table 6. FD and OM Scores

\begin{tabular}{llllll}
\hline Project 1 & \multicolumn{5}{l}{ Project 2} \\
\hline Date & FD Score & OM Score & Date & FD Score & OM Score \\
\hline 201606 & 12.62 & 82 & 201606 & 0.67 & 36 \\
201607 & 12.23 & 442 & 201607 & 0.51 & 150 \\
201608 & 10.56 & 290 & 201608 & 0.75 & 44 \\
201609 & 31.30 & 38 & 201609 & 1.07 & 32 \\
201610 & 28.59 & 42 & 201610 & 1.15 & 38 \\
201611 & 24.30 & 130 & 201611 & 1.22 & 54 \\
201612 & 25.01 & 52 & 201612 & -0.04 & 80 \\
201701 & 36.90 & 6 & 201701 & 2.69 & 6 \\
201703 & 29.71 & 56 & 201702 & 1.55 & 8 \\
201704 & 21.31 & 40 & 201703 & 2.01 & 42 \\
201705 & 27.77 & 48 & 201704 & 0.87 & 42 \\
201706 & 26.08 & 70 & 201705 & 1.89 & 60 \\
201707 & 18.93 & 58 & 201706 & 1.85 & 42 \\
201708 & 24.00 & 66 & 201707 & 1.84 & 38 \\
201709 & 12.75 & 106 & 201708 & 1.54 & 42 \\
201710 & -75.22 & 176 & 201709 & 2.67 & 48 \\
201711 & 18.21 & 362 & 201710 & 0.80 & 214 \\
201712 & -61.22 & 370 & 201711 & -1.01 & 128 \\
& & & 201712 & -0.94 & 300 \\
\hline
\end{tabular}

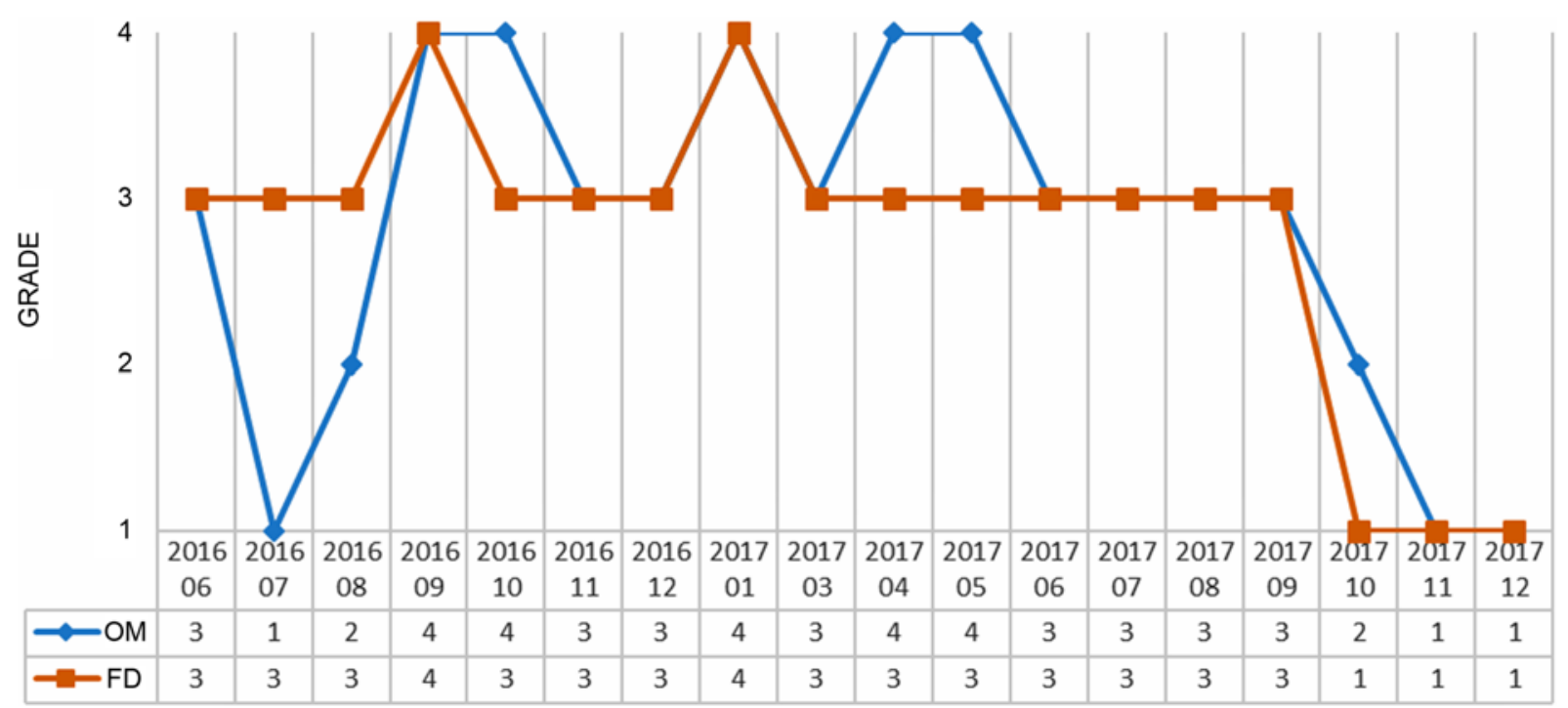

Fig. 4. Safety Degree of Project 1 Using OM and FD Approaches 


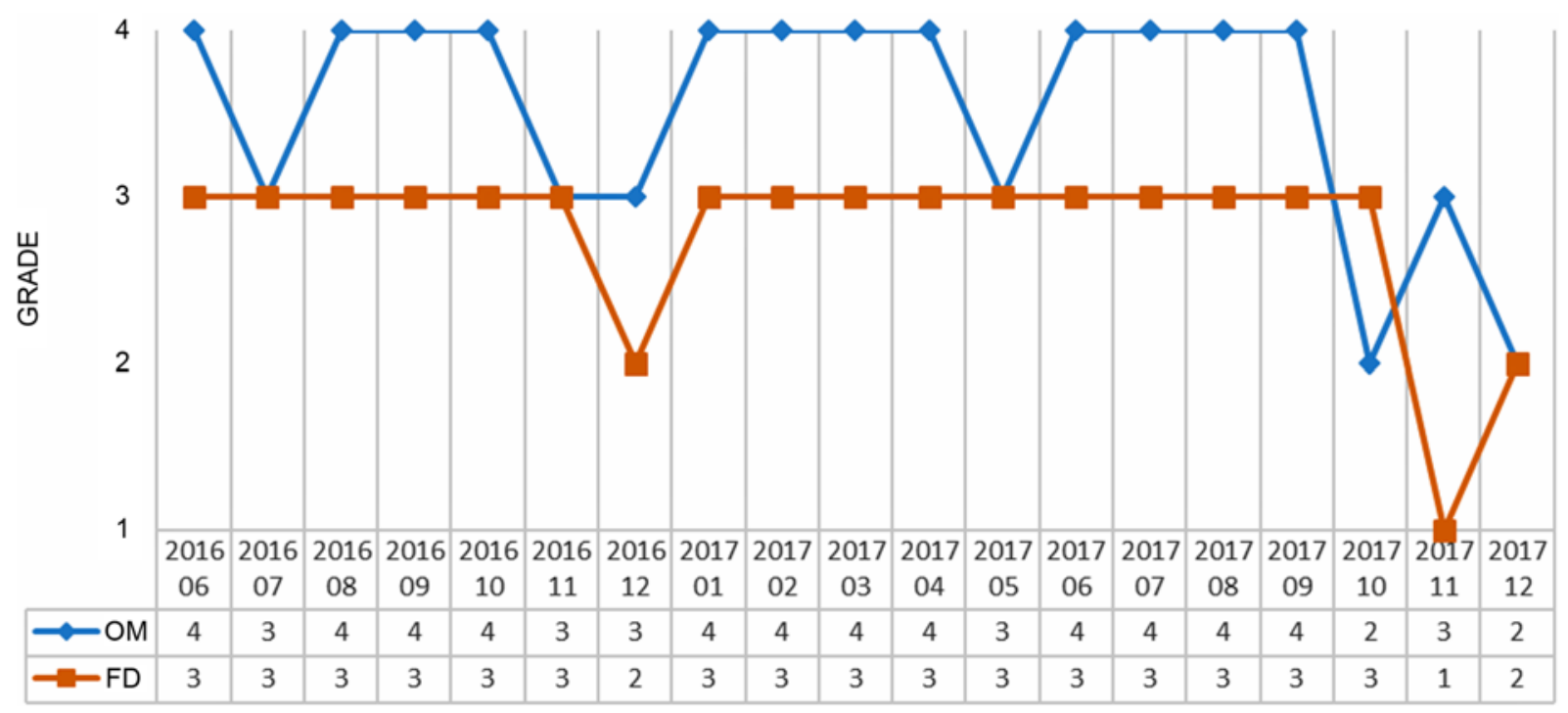

Fig. 5. Safety Degree of Project 2 Using OM and FD Approaches

indices generated using the proposed FD assessment method for the two case projects between June 2016 and December 2017. Overall, both projects were rated above "better" based on the $\mathrm{OM}$ approach. The FD approach was also aligned with the OM approach, but with a lower grade in its assessment for some periods of time in both projects.

As shown in Figs. 4 and 5, the results obtained by the FD approach are more conservative than the obtained by the OM, which seem to be crucial and rigorous from the perspective of construction safety assessment. The fluctuations of the safety grade were similar for the $\mathrm{OM}$ and $\mathrm{FD}$, with the construction safety performance tending to be in a better state (grade 3, grade 4 ) or in a poor state (grade 1, grade 2) simultaneously. When the OM grade was 4, the FD grade tended to be 3, indicating that the FD required higher safety standards to achieve the best assessment grade. For example, for 22 general hazards rectified on time in August 2016, the safety grade of the project 1 was 3 using the FM and 4 using the OM. For project 2 in September 2016, both methods provided a safety grade of 4 (best), with 19 general hazards rectified on time. Thus, the FD approach appears to be more conservative, with a lower tolerance for site hazards.

In the FD approach, the existence and elimination of hazards is a continuous process (Pereira et al., 2018). If the method only considered the hazards occurring within a certain assessment period (rectified on time or overdue rectification), this would neglect hazards that occurred in previous periods and continued to threaten the project in the assessment period due to rectification failure (Cooke, 2010; Han et al., 2014). These long-standing hazards seriously affect the safety of construction sites. Thus, by FD, the safety grade was 1 (worst), indicating that there were several unsafe factors in the construction site and/or the rectification efficiency of hazards was very low. In this situation, intervention may be required to rectify the site and prevent accidents caused by excessive hazards (Esmaeili et al., 2015; Lingard et al., 2017;
Pereira et al., 2018). Therefore, the results obtained by FD are more conservative and rigorous than those of the traditional methods of assessment.

This was further verified by the safety performance in September 2016 on the project 1 . In this period, there were 15 general hazards rectified on time and one general hazard classified as overdue rectification. The project safety grades were 4 and 3 using $\mathrm{OM}$ and $\mathrm{FD}$, respectively. Also, for periods with atypical differences in grading between methods (such as November 2017 for project 1 and July 2016 for project 2), the theoretical basis and data processing of the two methods are different. First, the risk consequence scores of the FD were extracted from industry standards (weighting of 3, 5, 10, or a maximum of 20); however, the scores from the OM were subjectively classified by experts, weighing up to 50 . This significantly influences the impact of the major hazards on the calculation results. For example, the hazard "there are no temporary protection measures for aerial work" was assigned 3 points according to the national standard criteria, whereas 50 points were given by the experts based on the OM. Through the FD calculation and its rectification coefficient, $\mu$, a more accurate safety performance index is determined during the safety assessment process. Considering the influence of rectification status, the OM weights "overdue rectification" the double of the weight for "rectification on time." However, the proposed FD approach uses a competitive corporation model to determine the weight of rectification on time, overdue rectification, and overdue non-rectification. In one case, OM identified two general hazards, two major hazards rectified on time, and six general hazards as overdue rectification. In contrast, FD indicated six hazards as rectified on time during the month (two that were discovered at the end of the month were classified as rectified on time because the correction occurred in the next month) and four hazards as overdue rectification (not eliminated until the following month). The OM calculation indicated grade 
3 (better); however, the FD calculation generated grade 1 (worst). Considering the failure to eliminate four hazards during this period and the risk of a long-term unsafe construction environment for workers, the FD result represents the actual situation.

Furthermore, as the construction safety environment danger increases, the FD grade is lower than the OM grade, which can provide an early warning of risk before the conditions further deteriorate or a significant incident occurs. Consider the example of a period in which eight general hazards and two major hazards were rectified on time, five general hazards and one major hazard were not rectified and classified as overdue rectification. The OM result for this period suggested that the safety grade was 2 (worse). However, the FD assessment also considered six hazards that occurred in the preceding month (September 2017), which were not rectified until mid-October, thus exceeding the allowable rectification time.

\subsection{Potential of Forecasting Tendencies}

By comparing the assessment results in the case studies, the proposed FD approach was found to be more conservative than the conventional approach. However, the FD approach can produce a better proactive warning ability than the conventional approach and has added benefits for the preliminary safety assessment. The proposed FD approach has potential for forecasting tendencies. For example, Fig. 4 shows that, for case project 1 between August 2016 and December 2016, both methods indicated a stable and safe condition for several months. Then, the FD grade decreased to 3 in October 2016, indicating the presence of some unsafe factors. The FD score continued to fall in November, when the OM index also began to fall. The OM grade tendency exhibited similar patterns to the FD grade, but appeared one month later than the FD approach. Further analysis showed that 19 hazards occurred and were rectified in September 2016, and the site was checked five times; however, in October 2016, the number of hazards had increased to 21 with only four safety checks. This shows that the safety status of the construction site had begun to deteriorate in that month, but only the FD assessment method could identify the change at that time.

This implies that when considering the dynamic safety measures performed by contractors, it helps in predicting on-site risks, and related proactive actions could be taken to prevent accidents (Hopkins, 2009; Guo and Yiu, 2016). The state of safety at a construction site incorporates the conservation of energy rather than only the occurrence of hazards, which has considered risk indicators, risk probability, and risk severity in dangerous situations. The proposed FD approach can be used to preliminarily assess and predict safety conditions after on-site safety inspection and hazard rectification by combining multiple safety-related measures (Esmaeili et al., 2015; Lingard et al., 2017) with dynamic characteristics of construction processes (Pereira et al., 2018) directly related to hazards. Conventional approaches did not consider dynamic changes (Cooke, 2010; Han et al., 2014; Xia et al., 2018) and therefore had a low predictive value to prevent accidents (Hopkins, 2009; Guo and Yiu, 2016).

\section{Conclusions}

This study has confirmed the feasibility of the FD approach for preliminary safety assessment in construction projects through its novel integration of safety conditions and contractor performance. The application of FD approach to construction safety considers the occurrence of hazards and rectification efficiency of management. Upon comparing the FD assessment with established methods, the FD approach was found to provide conservative empirical results and exhibited a proactive warning ability. In summary, the proposed FD approach theoretically contributes to a new perspective for the preliminary safety assessment under the dynamic nature of construction sites. Furthermore, the proposed method considers a comprehensive range of indicators and parameters, including rectification performance, number of project risks, inspection period, and construction area, which enables the comparison of safety performance between projects or assessment periods by independently changing parameters. Thus, the results of the safety assessment practically help in the development of proactive hazard control strategies and countermeasures in construction projects.

Despite the promise shown by the FD approach to safety assessment through its application in case studies, this research has some limitations. Further analysis is required if the FD approach to be adopted by broad site practitioners. Although the FD has the potential for warning on-site safety states, the risks are recognized to be complicated and highly stochastic, the FD approach should be supported with real-time evidence or on-site observations. There are definitive manners for measuring the pressure of the system in the FD approach; however, quantification of risk level remains subject to uncertainties. The factors which relates to the size of construction site, different construction teams, the role of corporate safety culture, safety climate and other aspects, have not been considered in the risk flow at this stage of framework development. These limitations will be considered in future studies.

\section{Acknowledgments}

This work was supported by the National Natural Science Foundation of China (grant number 51878382) and the Beijing General Municipal Engineering Design \& Research Institute Co., Ltd. (grant number 20202001333).

\section{ORCID}

Mei Liu ๑ http://orcid.org/0000-0002-0320-4688

Heap-Yih Chong ๑ http://orcid.org/0000-0002-6080-7530

Pin-Chao Liao ๑ http://orcid.org/0000-0001-6927-9204

\section{References}

Alruqi WM, Hallowell MR, Techera U (2018) Safety climate dimensions and their relationship to construction safety performance: A meta- 
analytic review. Safety Science 109:165-173, DOI: 10.1016/j.ssci. 2018.05.019

Ayhan BU, Tokdemir OB (2020) Accident analysis for construction safety using latent class clustering and artificial neural networks. Journal of Construction Engineering and Management 146(3):14, DOI: $10.1061 /$ (asce)co.1943-7862.0001762

Bartha P (2013) Analogy and analogical reasoning. The Stanford Encyclopedia of Philosophy, Retrieved June 25, 2013, https:// stanford.library.sydney.edu.au/archives/win2013/entries/abduction/

Christodoulou S (2010) Bid mark-up selection using artificial neural networks and an entropy metric. Engineering, Construction and Architectural Management 17(4):424-439, DOI: 10.1108/ 09699981011056600

Chritamara S, Ogunlana SO, Luong Bach N (2001) Investigating the effect of initial scope establishment on the performance of a project through system dynamics modelling. Engineering, Construction and Architectural Management 8(5-6):381-392, DOI: 10.1108/eb021198

Cooke DL (2010) A system dynamics analysis of the westray mine disaster. System Dynamics Review 19(2):139-166, DOI: 10.1002/ sdr. 268

Darrigol O, Frisch U (2008) From Newton's mechanics to Euler's equations. Physica D Nonlinear Phenomena 237(14):1855-1869, DOI: 10.1016/ j.physd.2007.08.003

Deng X, Zheng S, Xu P, Zhang X (2017) Study on dissipative structure of China's building energy service industry system based on brusselator model. Journal of Cleaner Production 150:112-122, DOI: 10.1016/j.jclepro.2017.02.198

Efthymiou K, Papakostas N, Mourtzis D, Chryssolouris G (2009) Fluid dynamics analogy to manufacturing systems. 42nd CIRP conference on manufacturing systems, Grenoble, France

Emmanuel G, Jean-Pierre C, Franck G (2016) A simplified approach to risk assessment based on system dynamics: An industrial case study. Risk Analysis 36(1), DOI: 10.1111/risa.12534

Esmaeili B, Hallowell MR, Rajagopalan B (2015) Attribute-based safety risk assessment. II: Predicting safety outcomes using generalized linear models. Journal of Construction Engineering and Management 141(8), DOI: 10.1061/(ASCE)CO.1943-7862.0000981

Fang DP, Huang XY, Hinze J (2004) Benchmarking studies on construction safety management in China. Journal of Construction Engineering And Management 130(3):424-432, DOI: 10.1061/(asce)0733-9364 (2004)130:3(424)

Feliciani C, Nishinari K (2018) Measurement of congestion and intrinsic risk in pedestrian crowds. Transportation Research Part C: Emerging Technologies 91:124-155, DOI: 10.1016/j.trc.2018.03.027

Feng Y, Zhang S, Wu P (2015) Factors influencing workplace accident costs of building projects. Safety Science 72:97-104, DOI: 10.1016/ j.ssci.2014.08.008

Gao T, Wang Q, Huang M, Wang X, Zhang Y (2018) A co-opetitive automated negotiation model for vertical allied enterprises teams and stakeholders. Entropy 20(4), DOI: 10.3390/e20040286

Grabowski M, Ayyalasomayajula P, Merrick J, Harrald JR, Roberts K (2007) Leading indicators of safety in virtual organizations. Safety Science 45(10):1013-1043, DOI: 10.1016/j.ssci.2006.09.007

Gunduz M (2018) Development of a safety performance index assessment tool by using a fuzzy structural equation model for construction sites. Automation in Construction 85:124-134, DOI: 10.1016/ j.autcon.2017.10.012

Guo BHW, Yiu TW (2016) Developing leading indicators to monitor the safety conditions of construction projects. Journal of Management in Engineering 32(1), DOI: 10.1061/(ASCE)ME.1943-5479.0000376
Guo CF, Zhang M, Devahastin S (2020) 3D extrusion-based printability evaluation of selected cereal grains by computational fluid dynamic simulation. Journal of Food Engineering 286:10, DOI: 10.1016/ j.jfoodeng.2020.110113

Hallowell MR, Bhandari S, Alruqi W (2020) Methods of safety prediction: Analysis and integration of risk assessment, leading indicators, precursor analysis, and safety climate. Construction Management and Economics 38(4):308-321, DOI: 10.1080/01446193.2019.1598566

Han SU, Saba F, Lee SH, Mohamed Y, Peña-Mora F (2014) Toward an understanding of the impact of production pressure on safety performance in construction operations. Accident Analysis and Prevention 68(1):106-116, DOI: 10.1016/j.aap.2013.10.007

Hinze J, Thurman S, Wehle A (2013) Leading indicators of construction safety performance. Safety Science 51(1):23-28, DOI: 10.1016/ j.ssci.2012.05.016

Hopkins A (2009) Thinking about process safety indicators. Safety Science 47(4):460-465, DOI: 10.1016/j.ssci.2007.12.006

Hosny O, Nassar K, Esmail Y (2013) Prequalification of Egyptian construction contractors using fuzzy-AHP models. Engineering, Construction and Architectural Management 20(4):381-405, DOI: 10.1108/ecam-09-2011-0088

Hou ZQ, Zeng YM (2016) Research on risk assessment technology of the major hazard in harbor engineering. Procedia Engineering 137: 843-848, DOI: 10.1016/j.proeng.2016.01.324

JGJ59-2011 (2011) Standard for construction safety inspection. Ministry of Housing and Urban Rural Development of People's Republic of China, Beijing, China

Jones TJ, Watts K, Whytock RC (2018) Using fluid dynamic concepts to estimate species movement rates in terrestrial landscapes. Ecological Indicators 93:344-350, DOI: 10.1016/j.ecolind.2018.05.005

Kachani S, Perakis G (2006) Fluid dynamics models and their applications in transportation and pricing. European Journal of Operational Research 170(2):496-517, DOI: 10.1016/j.ejor.2004.07.047

Kamardeen I (2009) Strategic safety management information system for building projects in Singapore. Engineering, Construction and Architectural Management 16(1):8-25, DOI: 10.1108/ 09699980910927868

Kumar K, Garg H (2016) TOPSIS method based on the connection number of set pair analysis under interval-valued intuitionistic fuzzy set environment. Computational and Applied Mathematics 37(2): 1319-1329, DOI: 10.1007/s40314-016-0402-0

Li X, Wang K, Liu L, Xin J, Yang H, Gao C (2011) Application of the entropy weight and TOPSIS method in safety evaluation of coal mines. Procedia Engineering 26(4):2085-2091, DOI: 10.1016/ j.proeng.2011.11.2410

Li A, Zhao Z (2017) Crane safety assessment method based on entropy and cumulative prospect theory. Entropy 19(1):44, DOI: 10.3390/ e19010044

Lighthill MJ, Whitham GB (1955) On kinematic waves. II. A theory of traffic flow on long crowded roads. Proceedings of the Royal Society A: Mathematical Physical \& Engineering Sciences 229(1178):317345, DOI: 10.1098/rspa.1955.0089

Lingard H, Hallowell M, Salas R, Pirzadeh P (2017) Leading or lagging? Temporal analysis of safety indicators on a large infrastructure construction project. Safety Science 91:206-220, DOI: 10.1016/ j.ssci.2016.08.020

Long DN, Dai QT, Chandrawinata MP (2016) Predicting safety risk of working at heights using bayesian networks. Journal of Construction Engineering \& Management 142(9):1-16, DOI: 10.1061/(ASCE) CO.1943-7862.0001154 
López-Alonso M, Ibarrondo-Dávila MP, Rubio-Gámez MC, Munoz TG (2013) The impact of health and safety investment on construction company costs. Safety Science 60(12):151-159, DOI: 10.1016/ j.ssci.2013.06.013

Ma J, Chan CK, Ye Z, Zhu Z (2018) Effects of maximum relaxation in viscoelastic traffic flow modeling. Transportation Research Part B: Methodological 113:143-163, DOI: 10.1016/j.trb.2018.05.013

Mahmoud S, Zayed T, Fahmy M (2019) Development of sustainability assessment tool for existing buildings. Sustainable Cities and Society 44:99-119, DOI: 10.1016/j.scs.2018.09.024

Manning FST, Richard E (1991) Oilfield processing of petroleum: Natural gas. PennWell Books, Tulsa, OK, USA

Mishra VK, Singh SK, Patel SP, Pandey S, Dwivedi N, Dwivedi S (2015) Applications of fluid mechanics in different engineering fields. Online Journal of Biosciences and Informatics 1(1)

Mohamed S, Chinda T (2011) System dynamics modelling of construction safety culture. Engineering Construction \& Architectural Management 18(3):266-281, DOI: 10.1108/09699981111126179

Mohammadi A, Tavakolan M, Khosravi Y (2018) Factors influencing safety performance on construction projects: A review. Safety Science 109:382-397, DOI: 10.1016/j.ssci.2018.06.017

Nima K, Faisal K, Paul A, Valerio C (2014) Risk management of domino effects considering dynamic consequence analysis. Risk Analysis 34(6):1128-1138, DOI: 10.1111/risa.12158

Peng G (2013) A new lattice model of two-lane traffic flow with the consideration of optimal current difference. Communications in Nonlinear Science and Numerical Simulation 18(3):559-566, DOI: 10.1016/j.cnsns.2012.07.015

Pereira E, Han S, Abourizk S (2018) Integrating case-based reasoning and simulation modeling for testing strategies to control safety performance. Journal of Computing in Civil Engineering 32(6), DOI: 10.1061/(ASCE)CP.1943-5487.0000792

Qiu G, Song R, He S, Yin W (2018) The pedestrian flow characteristics of Y-shaped channel. Physica A: Statistical Mechanics and its Applications 508:199-212, DOI: 10.1016/j.physa.2018.05.015

Rajendran S (2013) Enhancing construction worker safety performance using leading indicators. Practice Periodical on Structural Design and Construction 18(1):45-51, DOI: 10.1061/(asce)sc.1943-5576. 0000137

Richards PI (1956) Shock waves on the highway. Operations Research 4:42-51, DOI: 10.1287/opre.4.1.42

Robson LS, Ibrahim S, Hogg-Johnson S, Steenstra IA, Van Eerd D, Amick BCI (2017) Developing leading indicators from ohs management audit data: Determining the measurement properties of audit data from the field. Journal of Safety Research 61:93-103, DOI: 10.1016/ j.jsr.2017.02.008

Rubio-Romero JC, Pardo-Ferreira MdC, De la Varga-Salto J, GalindoReyes F (2018) Composite leading indicator to assess the resilience engineering in occupational health \& safety in municipal solid waste management companies. Safety Science 108:161-172, DOI: 10.1016/j.ssci.2018.04.014

Seminara G, Carli B, Forni G, Fuzzi S, Mazzino A, Rinaldo A (2020) Biological fluid dynamics of airborne COVID-19 infection. Rendiconti Lincei-Scienze Fisiche E Naturali 31(3):505-537, DOI: 10.1007/ s12210-020-00938-2

Shea T, De Cieri H, Donohue R, Cooper B, Sheehan C (2016) Leading indicators of occupational health and safety: An employee and workplace level validation study. Safety Science 85: 293-304, DOI: 10.1016/j.ssci.2016.01.015

Sheehan C, Donohue R, Shea T, Cooper B, Cieri HD (2016) Leading and lagging indicators of occupational health and safety: The moderating role of safety leadership. Accident Analysis \& Prevention 92:130-138, DOI: 10.1016/j.aap.2016.03.018

Sun Y (2018) Kinetic Monte Carlo simulations of two-dimensional pedestrian flow models. Physica A: Statistical Mechanics and its Applications 505:836-847, DOI: 10.1016/j.physa.2018.04.017

Thurston E, Glendon AI (2018) Association of risk exposure, organizational identification, and empowerment, with safety participation, intention to quit, and absenteeism. Safety Science 105:212-221, DOI: 10.1016/ j.ssci.2018.02.012

Tosin A, Zanella M (2019) Kinetic-controlled hydrodynamics for traffic models with driver-assist vehicles. Multiscale Modeling \& Simulation 17(2):716-749, DOI: 10.1137/18m1203766

Versteeg K, Bigelow P, Dale AM, Chaurasia A (2019) Utilizing construction safety leading and lagging indicators to measure project safety performance: A case study. Safety Science 120:411-421, DOI: 10.1016/j.ssci.2019.06.035

Wang F, Deng FJ, Wang YP (2020) Construction method and application of real-time monitoring and early-warning model for anaerobic reactor leakage. Process Safety Progress 11, DOI: 10.1002/prs.12144

Wiendahl HH, Roth N, Westkämper E (2002) Logistical positioning in a turbulent environment. CIRP Annals - Manufacturing Technology 51(1):383-386, DOI: 10.1016/S0007-8506(07)61542-X

Xia N, Zou PXW, Liu X, Wang X, Zhu R (2018) A hybrid BN-HFACS model for predicting safety performance in construction projects. Safety Science 101:332-343, DOI: 10.1016/j.ssci.2017.09.025

Yu Y, Darko A, Chan APC, Chen C, Bao F (2018) Evaluation and ranking of risk factors in transnational public-private partnerships projects: Case study based on the intuitionistic fuzzy analytic hierarchy process. Journal of Infrastructure Systems 24(4), DOI: 10.1061/ (ASCE)IS.1943-555X.0000448

Zhang X (2011) Social risks for international players in the construction market: A China study. Habitat International 35(3):514-519, DOI: 10.1016/j.habitatint.2011.02.005

Zhao H, Zhang G, Li W, Gu T, Zhou D (2018) Lattice hydrodynamic modeling of traffic flow with consideration of historical current integration effect. Physica A: Statistical Mechanics and its Applications 503:1204-1211, DOI: 10.1016/j.physa.2018.08.072 